\title{
Detection and Separation of Eeg Artifacts Using Wavelet Transform
}

\author{
R. Suresh Kumar ${ }^{1}$, P.Manimegalai ${ }^{2}$ \\ ${ }^{1}$ Research Scholar/Department of ECE, Karpagam Academy of Higher Education, Coimbatore, India. \\ ${ }^{2}$ Professor/Department of ECE, Karpagam Academy of Higher Education, Coimbatore, India.
}

\begin{tabular}{l}
\hline \hline Article Info \\
\hline Article history: \\
Received Jun 3, 2018 \\
Revised Jul 28, 2018 \\
Accepted Aug 13, 2018 \\
\hline
\end{tabular}

\section{Keywords:}

Electroencephalography

Mean square error

Signal to noise ratio

Wavelet transform

\begin{abstract}
Bio-medical signal processing is one of the most important techniques of multichannel sensor network and it has a substantial concentration in medical application. However, the real-time and recorded signals in multisensory instruments contains different and huge amount of noise, and great work has been completed in developing most favorable structures for estimating the signal source from the noisy signal in multichannel observations. Methods have been developed to obtain the optimal linear estimation of the output signal through the Wide-Sense-Stationary (WSS) process with the help of time-invariant filters. In this process, the input signal and the noise signal are assumed to achieve the linear output signal. During the process, the nonstationary signals arise in the bio-medical signal processing in addition to it there is no effective structure to deal with them. Wavelets transform has been proved to be the efficient tool for handling the non-stationary signals, but wavelet provide any possible way to approach multichannel signal processing. Based on the basic structure of linear estimation of nonstationary multichannel data and statistical models of spatial signal coherence acquire through the wavelet transform in multichannel estimation. The above methods can be used for Electroencephalography (EEG) signal denoising through the original signal and then implement the noise reduction technique to evaluate their performance such as SNR, MSE and computation time.
\end{abstract}

Copyright (c) 2018 Institute of Advanced Engineering and Science. All rights reserved.

\section{Corresponding Author:}

R. Suresh Kumar

Research Scholar/Department of ECE,

Karpagam Academy of Higher Education,

Coimbatore, India.

Email: arsksuresh@gmail.com

\section{INTRODUCTION}

Brain is the most important organ in human beings, for controlling the coordination of human muscles and nerves. Human brain is made up of millions of neurons and it is a connected nervous system. The electrical activity of the brain and the language of communication with the nervous system are called as Electroencephalography (EEG) signals. The neurons move in human brain while processing information, by changing the flow of electrical currents across their membranes. The neurons movement is observed through electrodes placed on the scalp and the signal is analyzed with respect to change in the electrical properties. Activity of the brain writing process is amplified and recorded from the potentials between different electrodes in Electroencephalogram (EEG) [1]. The recording is used to investigate the neuron movement of human brain commonly through one EEG. It can be used to evaluate the people having brain disorders and also EEG is used to determine brain death. The continuous signal analysis is more complex. The EEG signal are categorized in different wave length namely Alpha $(\alpha)$ waves $(7.5-14 \mathrm{~Hz})$, Beta $(\beta)$ waves $(14-40 \mathrm{~Hz})$, Gamma $(\gamma)$ waves (above $40 \mathrm{~Hz}$ ), Theta $(\Theta)$ waves $(4-7.5 \mathrm{~Hz})$, Delta $(\Delta)$ waves $(0.5-4 \mathrm{~Hz})[2-4]$. All the waves represent different movement of neurons in human brain. 
The EEG signals have very small amplitude $(\mathrm{mV})$ so the noise occurs easily or the original signal gets contaminated. During the recording or observation of the EEG signal, various types of noises occur such as noise from baseline movement, EMG disturbance, and human body and from other external sources. In EEG signal these types of noise signals are called artifacts and this noise need to be removed from the original EEG signal during the analysis of brain related diseases.

Moreover, during the EEG recording the original signal is also affected by other unknown random signals which can be modeled as additive random noise. These occurrences complicate the analysis and interpretation of the EEGs, and the first important processing step would be the elimination of the artifacts and noise. Our goal is to contribute to EEG artifact rejection by proposing an original and more complete automatic methodology consisting if optimized combination of several signal processing and data analysis techniques. There are so many denoising techniques employed to remove the artifacts from the EEG original signal. Some of the denoising techniques used to remove the noises are ICA denoising, PCA denoising, Wavelet based denoising. The above said techniques employed for denoising the EEG signal and their performance can be evaluated by measuring the parameters like SNR, MSE and computation time etc.

This paper is organized as follows: The second section presents a brief history about the EEG signal artifacts and noises rejection presented in the literature. The third section contains the proposed approach for denoising. In this part the wavelet denoising is briefly explained and the methodological steps involved to acquire the noise rejected output signal in a proposed wavelet transform. The fourth section shows the simulation result of the proposed technique in Matlab and VLSI.

\section{RELATED WORK}

In bio-medical signal process, artifacts are unwanted noise caused by the original physiological affair based on the interest. Thus, the aim of analysis depending on the decision should be made as identifying the original and artifact signal. For reliable analysis artifacts classification should be considered, which if ignored, might considerably influence the results and therefore the resulting conclusions. EEGs are typically recorded in conjunction with different physiological signals which can interfere with the exact EEGs. Such artifact like ocular, muscle, electrical field changes, transmission line interference, movements of head and electrodes.

Figure.1 illustrates sections of graphical record contaminated by typical samples of artifacts. Once some of knowledge corrupted by an artifact has been with successfully known then there are different ways which may be adopted, betting on the shape of that artifact. In extreme cases the whole epoch that contains the artifact might have to be discarded, various normal artifact detector is used and therefore the signals that is contaminated by the artifact is known and discarded. As an alternative, in some instances, there is a potential to estimate the original EEG signal using appropriate signal process techniques by suppressing the artifact.

The effortless way to eliminate artifacts is to find at the period of occurrence and recorded data is eliminated. One technique that is used to take artifact as any important deviation from the traditional is to permit detection by seeking variations (non- stationaries) within the measured signal. This technique should be applied with care, since the Original EEG is itself non stationary, therefore major parameters have to be designated, so the entire connected non-stationaries are detected. Energy operators may be helpful markers of sudden changes (e.g. spikes) as they are sensitive to instant fluctuations [5] however will require more sensitivity to record accurate changes within the signal spectrum.

A set of mathematical functions that transforms number of correlated variables into a smaller number of unrelated variables is known as Principal Component. The Principal Component Analysis (PCA) is a primary element that accounts for the maximum amount of the variability within the data as possible, and every succeeding element accounts for the maximum amount of the remaining variability as potential. Principal components are absolute and independent to provide the recording dataset that is normally circulated. PCA is more accurate to the relative level of the initial variables[6-9]. Depending on the application, it is additionally named as distinct Karhunen-Loève transform, the Hotelling transform or Proper orthogonal decomposition.

The mathematical method utilized in PCA is termed as Eigen analysis. It tends to solve the Eigen values and eigenvector of a square symmetric matrix with sum of square and cross product. The eigenvector is related to the biggest Eigen values and has constant direction because it is the first principal element. The eigenvector related to the second largest Eigen values determine the direction of the second principal component. The addition of the Eigen values equals the trace of the matrix and therefore the most numbers of eigenvectors equals the quantity of rows (or columns) of this matrix.[10-11]. An alternative approach is obtained using ICA (Independent Component Analysis) that makes the multichannel character of most EEG record signals to decompose the information into a collection of random variables that are maximally

IJ-ICT Vol. 7, No. 3, December 2018: 149- 156 
independent[12]. This decomposition is thought to be representing the supply signals that underlie in the measured data set. The magnetism nature of EEG signals implies that these signals fulfill the assumptions of the ICA algorithmic rule, i.e. ones supported by a right away mixture model. Consequently EEG analysis normally has been a fruitful application space for ICA [13-18].

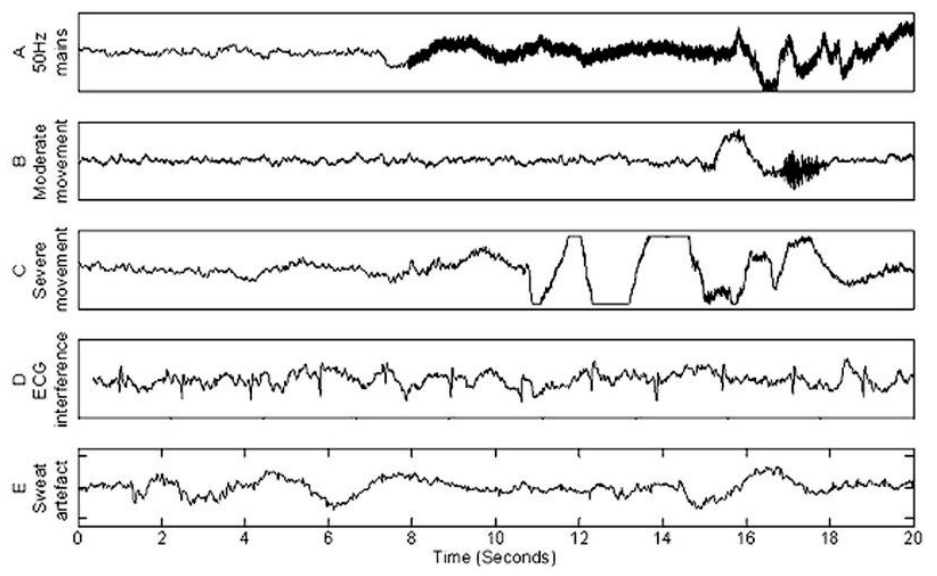

Figure 1. EEG artifacts (A) $50 \mathrm{~Hz}$ mains interference waves on the EEGs. (B) Neuron movement causes from the EEG (C) Movement that can clip the EEG. (D) ECG interference appears as a pulsed EEG (E) baseline EEG

The ways rely on the assumption that the artifact and EEG may arise from completely different (statistically independent) sources. Therefore by estimating those supply parts one can, determine that parts relate to the artifact and it relates to the EEG Signal. This permits the reconstruction of the sensing element signals mistreatment, solely the EEG signal connected sources and thus eliminating the artifact. A key drawback with ICA is that the study and automatic identification/separation of parts with reference to graphical record and artifact. Further, if the signal is temporally metameric then the ICA decomposition lacks consistency between sections and care must be exercised once recombining the information across segment boundaries [19-22].

\section{WAVELET DENOISING}

In this section, it is explained the results of research and at the same time is given the comprehensive discussion. Results can be presented in Figures, graphs, Tables and others that make the reader understand easily [2], [23]. The discussion can be made in several sub-chapters.

The implementation of Wavelet Transform (WT) time-frequency representation helps to present the frequency and time in sequence with respect to time. In time-frequency representation the time domain signal is passed through two different filters namely low pass and high pass filters, here both portion are considered. The filters are used to eliminate the artifacts and noise signals from original signal and the process is called as decomposition. The operation is continuously repeated until the signal is decomposed to a cutoff level or to a certain level. A group of different frequency bands obtained from the EEG recording signals representing in the same. The high frequency components are enhanced to improve with respect to time and the low frequency components are enhanced to determine the frequency. The wavelet transform is defined as equation 1 .

$$
W(a, b)=\int_{-\infty}^{\infty} x(t) \frac{1}{\sqrt{a}} \psi\left(\frac{t-b}{a}\right) d t
$$

The above equation is said to be a mother wavelet transform. The equation contains two different variables $(a, b)$ used to transform the signals. Both variables are representing translation and scaling parameters and the denoising function is obtained through the mother wavelet transform. The low frequency components are matched with detailed view and the high frequency components are matched with non- 
detailed view. In scaling process either the input signal is to be compressed or expanded. The high frequency components (large signals) are always stretched out and low frequency components are compressed.

During the denoising process the synthesis and analysis of original signal and sufficient information is obtained through the Discrete Wavelet Transform (DWT) and it provides the maximum reduction with minimum computation time. The different frequency bands of input signals are analyzed with different resolution that is used to decompose the signals to get detailed information. The DWT functions are same in the mother wavelet transform associated with low pass and high pass filters in time domain signals. The original input signal $\mathrm{x}[\mathrm{n}]$ is first processed through a half-band high-pass filter $\mathrm{g}[\mathrm{n}]$ and a low-pass filter $\mathrm{h}[\mathrm{n}]$. The signal can be sub-sampled by dividing it by 2 , simply by discarding every other sample. This constitutes one level of decomposition and can be expressed as follows equation $2 \& 3$ :

$$
\begin{aligned}
& Y_{\text {high }}[k]=\sum_{n} x[n] \cdot g[2 k-n] \\
& Y_{\text {high }}[k]=\sum_{n} x[n] \cdot h[2 k-n]
\end{aligned}
$$

Where, yhigh $[\mathrm{k}]$ and ylow[k] are the output of the high pass and low pass filters after the subsampling divided by 2 . These decomposition techniques have time resolution and only half the number of samples describes the entire signal.

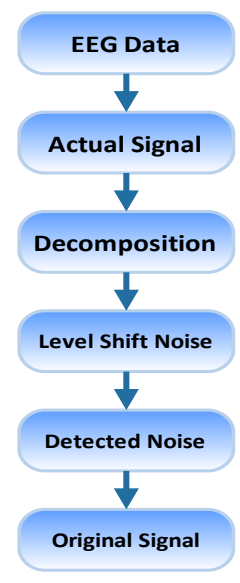

Figure 2. Block diagram of proposed system EEG data decomposition level shift noise detected noiseoriginal signal

However, this procedure doubles the frequency resolution, because the half of the frequency is spanned effectively by reducing the uncertainty in the frequency band and repeats the second half of the frequency band to reduce the noise. The above procedure is also called as sub-band coding and it can be frequently used for additional decomposition. At each level sub-sampling filtering results is obtained with half the frequency band span. The following statistical features were utilized to represent the time-frequency distribution of the EMG signals: Standard Deviation, Mean, Average power, and ratio of absolute mean of the coefficients in each sub-band samples.

\section{RESULTS}

The research study was to find out the possibility of minimizing the artifacts and noises in EEG signal using wavelet denoising technique. The main objective of the study was to minimize the artifacts and noise level without affecting the original EEG signal. Since the EEG recording requires clinical information from many channels (standard channels 32 - 44), the input sources high so a critical design is used during the analysis using real time multichannel artifacts and noise detection system. The result of the study currently shows that the utilization of the WT significantly decreases the input size, lacking in performance while not maximum compromise on the performance. The foremost vital consideration of performance was the correct choice of the scale in the input signal. Wavelets were found appropriate for analysis as a result of the 
compact support. Programs are developed to show the sample waveforms for various channels and also for decomposition of the undulation mistreatment DB-1 \& DB-5 in addition the original signal is denoised and compressed into waveforms for every channel. The sampled outputs of first high-pass and low-pass filters provide the detail D1-D5. We have extracted 6 different values from the frequency bands to calculate D1, D2, D3, D4, D5 and A5 of DWT. Figure 3 shows the extracted output wave form of the DWT and the Figure 4 shows the input noise signal and output signal obtained from the wavelet transform. In this Figures the 4097 input samples are taken to extract the different resolution in wavelet transform.

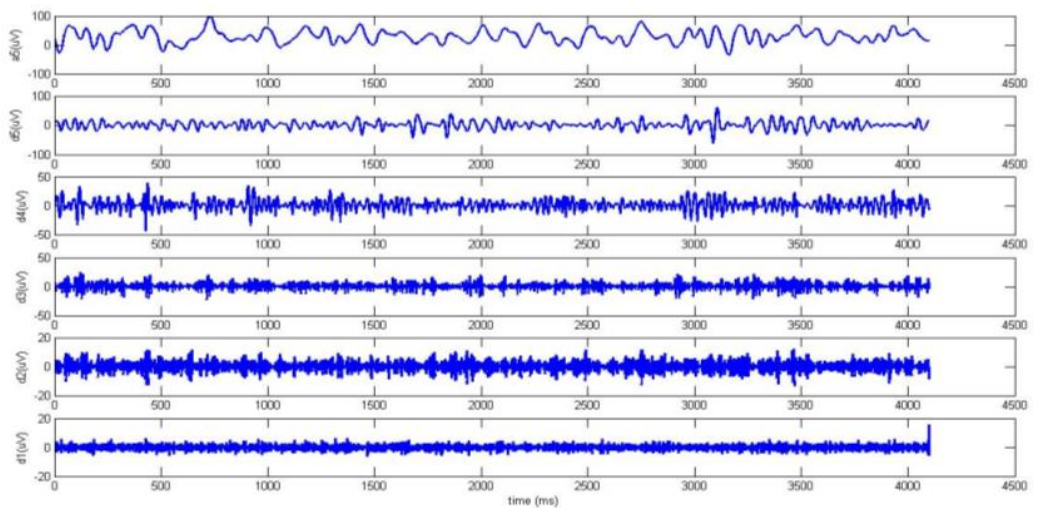

Figure 3. Decomposition waves of D1 to D5 and A5
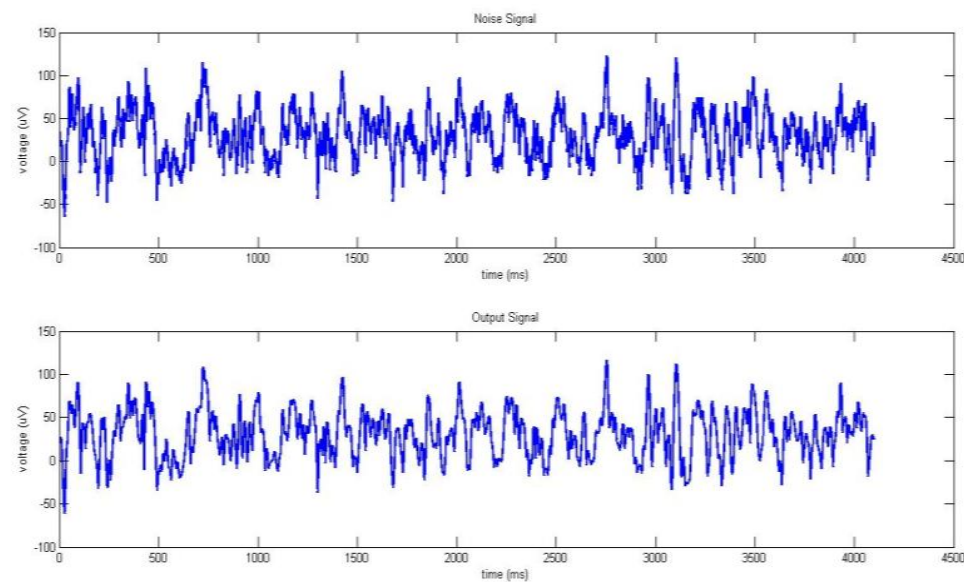

Figure 4. Shows the input noise signal and the corresponding output signal from DWT

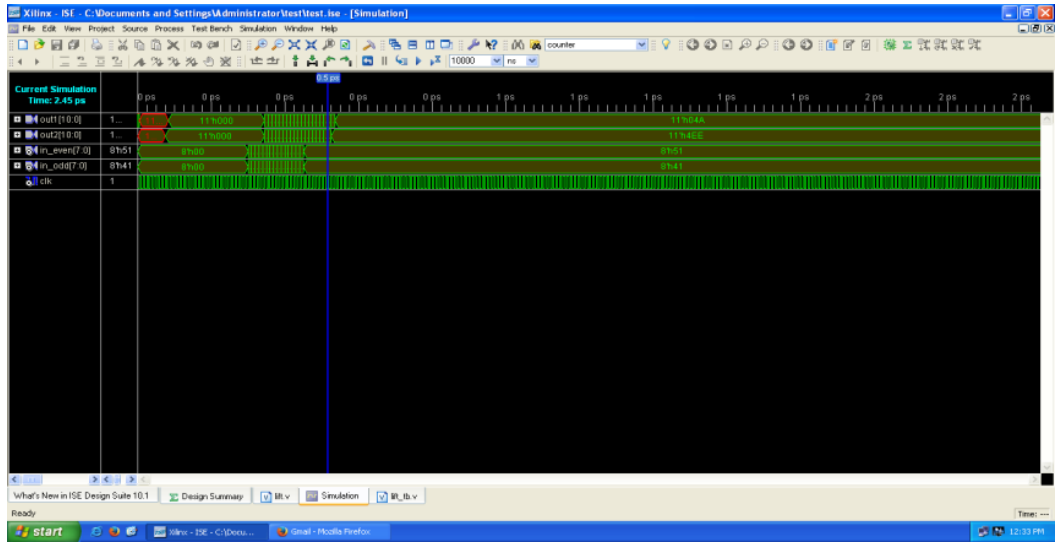

Figure 5. Simulated output waveform of wavelet transform 
In Figure 5 shows the output waveform of the DWT are obtained from the different wave signals at different resolution on the fundamental signals alpha, gama, beta and deta waves and the HDL to RTL view are shown in Figure 6.

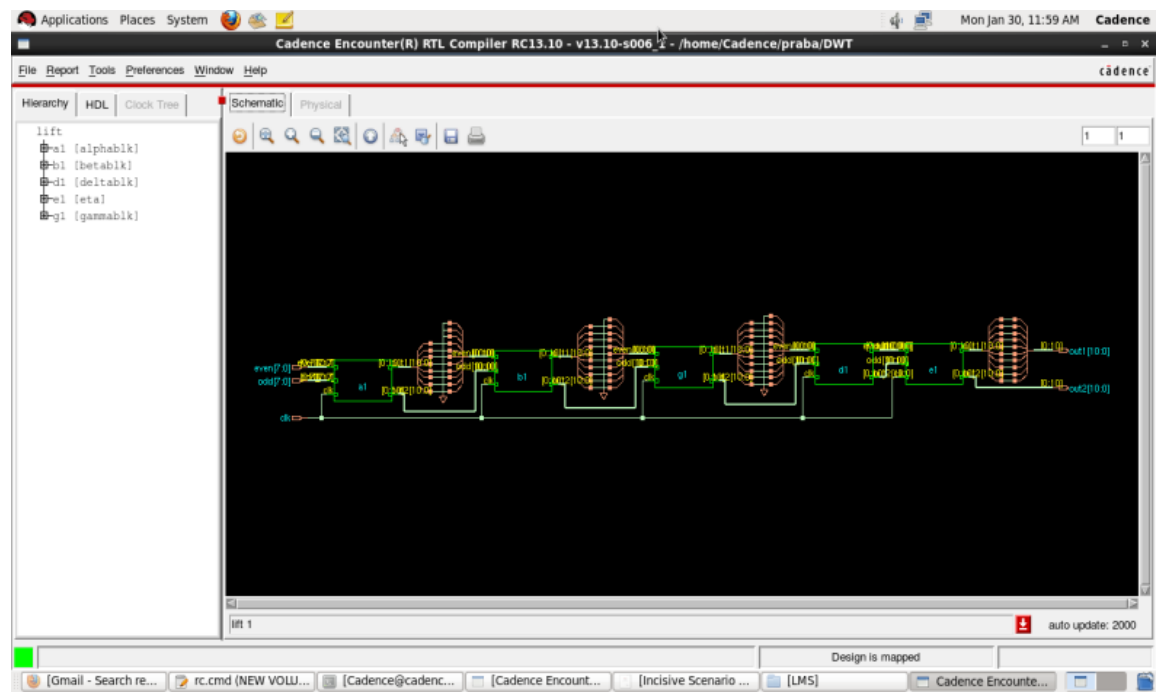

Figure 6. RTL view of wavelet transform

From Table 1 shows the area utilized with respect to the no of cells for DWT based denoising architecture and the corresponding graphical representation are shown in Figure 7. In Table 2 shows the power utilization of DWT architecture and the graphical representation shown in Figure 8 and the Table 3 contains time utilized for DWT architecture and Figure 9 shows the graphical representation. The above result was obtained in TMSE $90 \mathrm{~nm}$ technology Cadence Environment.

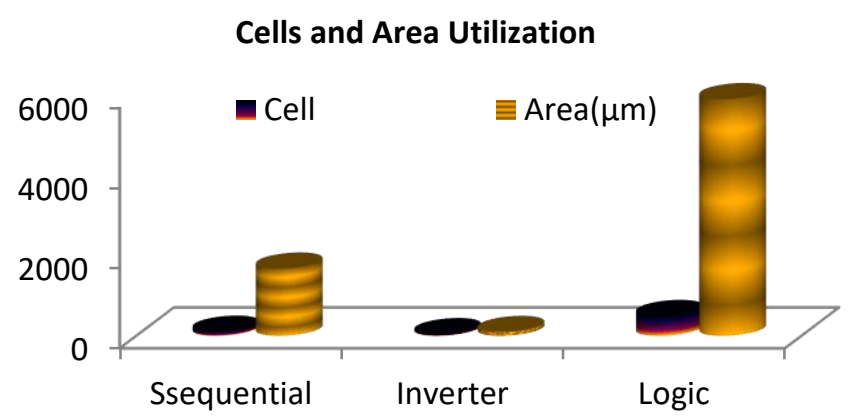

Figure 7. No of cells and area utilized for DWT based denoising

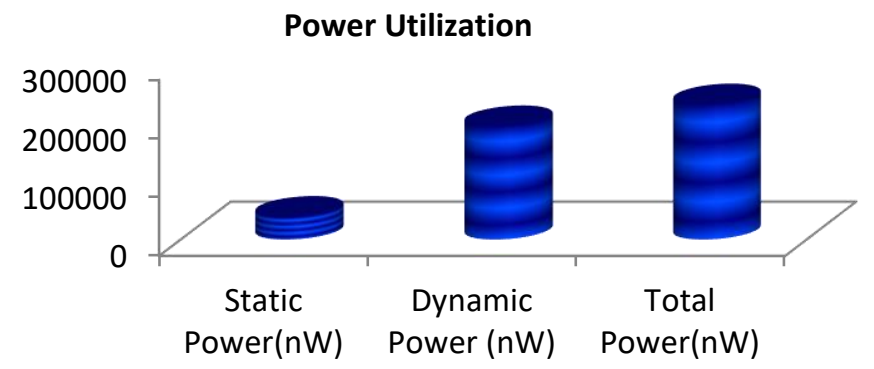

Figure 8. Power utilization of DWT based denoising 


\section{Time Utilization}

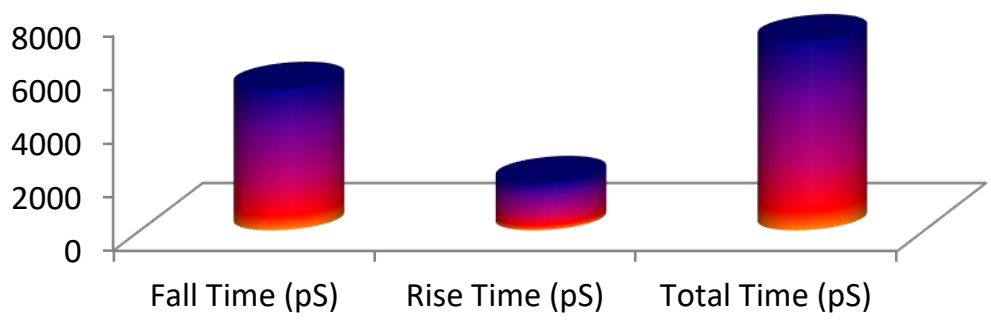

Figure 9. Time utilization of DWT based denoising

Table 1. Cell and Area Utilization of DWT based Denoising Architecture

\begin{tabular}{ccc}
\hline \multicolumn{3}{c}{ Cell and Area Utilization } \\
\hline ANC & Cell & Area $(\mu \mathrm{m})$ \\
Ssequential & 104 & 1685 \\
Inverter & 43 & 98 \\
Logic & 498 & 5911 \\
Total & 645 & 7694 \\
\hline
\end{tabular}

Table 2. Power Utilization of DWT based Denoising Architecture

\begin{tabular}{cccc}
\hline \multicolumn{3}{c}{ Power Utilization } \\
\hline Cells & Static Power (nW) & Dynamic Power (nW) & Total Power (nW) \\
645 & 37397 & 191928 & 229325 \\
\hline
\end{tabular}

Table 3. Time Utilization of DWT based Denoising Architecture

\begin{tabular}{ccc}
\hline & Time Utilization & \\
\hline Fall Time $(\mathrm{pS})$ & Rise Time $(\mathrm{pS})$ & Total Time $(\mathrm{pS})$ \\
5276 & 1772 & 7048 \\
\hline
\end{tabular}

\section{CONCLUSION}

Wavelets function is a robust tool used for the task of signal denoising. The ability to decompose a signal into a totally different scale is extremely vital for denoising, and it improves the analysis of the signal considerably. A real-time denoising VLSI based wavelet transform technique is employed in this paper to remove the artifacts and power-line interferences from EEG signal. Wavelet functions square measure is used for denoising. The results of denoising is evaluated using SNR, RMSE and computation time From the Table 1 it is clear that the initial signal and db5 denoised signal square measure additional correlative and shows higher results compared to alternative wavelets employed in denoising the EEG signal. So it will be used for analyzing EEG signal. Careful analysis of the graphical record signals will provide data associated to patients affected with brain disorders. In future, additional advanced ways will be explored for denoising of the EEG signal corrupted by alternative sources of interference. Additionally these method are going to be implemented and tested on real EEG signals. The implementation and prototyping of the proposed technique used Verilog HDL in Cadence 45nm environment.

\section{REFERENCES}

[1]. S.Y. Tseng, R.C. Chen, F.C. Chong, T.S. Kuo, Evaluation of parametric methods in EEG signal analysis, Medical Engineering \& Physics 17 (1995) 71-78.

[2]. GiuseppinaInuso ,Wavelet-ICA methodology for efficient artifact removal from Electroencephalographic recordings, Proceedings of International Joint Conference on Neural Networks, Orlando, Florida, USA, August 1217, 2007.

[3]. Mrs V Baby Deepa ,Dr P Thangaraj, Dr S Chitra, Investigating the performance improvement by sampling techniques in EEG data, International Journal on Computer Science and Engineering , Vol. 2,Jun.2010,pp:20252028.

[4]. Dong Kang; LuoZhizeng ,A Method of Denoising Multi-channel EEG Signals Fast Based on PCA and DEBSS Algorithm, Computer Science and Electronics Engineering (ICCSEE), 2012 International Conference on , vol.3, no., pp.322-326, 23-25 March 2012. 
[5]. Arvaneh M, Guan C, Ang K, Quek H (2011) Optimizing the channel selection and classification accuracy in eegbased bci. IEEE Transactions on Biomedical Engi-neering, 58:1865-1873.

[6]. Balli T, Palaniappan R (2010) Classification of biological signals using linear and nonlinear features. Physiological measurement 31(7):903.

[7]. Bashashati A, Fatourechi M, Ward RK, Birch GE (2007) A survey of signal pro-cessing algorithms in braincomputer interfaces based on electrical brain signals. Journal of Neural engineering, 4(2):R35-57.

[8]. P. Hae-Jeong, J. Do-Un, P. Kwang-Suk, Automated detection and elimination of periodic ECG artifacts in EEG using the energy interval histogram method,IEEE Trans. Biomed. Eng. 49 (2002) 1526-1533

[9]. J.S. Barlow, Methods of analysis of non-stationary EEGs, with emphasis on segmentation techniques - a comparative review, J. Clin. Neurophysiol. 2 (1985) 267-304

[10]. P. Ashok Babu1, Removal of ocular artifacts from EEG signals using adaptive threshold PCA and Wavelet Transforms, International Journal of Electronic Signals and Systems, vol 1,2012

[11]. Janett Walters-Williams \& Yan Li, A New Approach to Denoising EEG Signals - Merger of Translation Invariant Wavelet and ICA, International Journal of Biometrics and Bioinformatics, Volume (5) : Issue (2) : 2011

[12]. GiuseppinaInuso, Wavelet-ICA methodology for efficient artifact removal from Electroencephalographic recordings, Proceedings of International Joint Conference on Neural Networks, Orlando, Florida, USA, August 1217, 2007.

[13]. M. E. Davies, C. J. James, Source separation using single channel ICA Signal Processing. 2007; 87: 1819-1832

[14]. C.K. Chui, An Introduction to Wavelets, Academic Press, New York, 1992, Chapter 5, pp. 158-160.

[15]. O. Rioul, M. Vetterli, Wavelets and Signal Processing, IEEE Signal Process. Mag. 8 (4) (1991) 14-38

[16]. Unser M, Aldroubi A, A Review of Wavelets In Biomedical Applications, Proceedigs of the IEEE 84(4): 626-638, 1999.

[17]. S. Mallat, A theory for multiresolution signal decomposition: the wavelet representation, IEEE Trans. Pattern Anal. Machine Intell. 11 (7) (1989) 674-693.

[18]. M. S. Crouse, R. D. Nowak, and R. G. Baraniuk, Wavelet-based statistical signal processing using hidden Markov models,IEEE Trans. Signal Process, vol. 46, no. 4, pp. 886-902, 1998.

[19]. Lotte F, Congedo M, L' ecuyer A, Lamarche F, Arnaldi B (2007) A review of classi-fication algorithms for EEGbased brain-computer interfaces. Journal of Neural Engineering, 4:R1-R13.

[20]. Juha Karhunen, JyrkiJoutsens, Generalization of principal component analysis optimization problems and Neural Network, Vol. 8,pp:549-562,March.1995.

[21]. Kachenoura A, Albera L, Senhadji L, Comon P (2008) ICA: A potential tool for BCI systems. IEEE Signal Processing Magazine, 25(1):57-68

[22]. Vigario R., Jousmaki V., Hamalainen M., Hari R., and Oja E,Independent component analysis for identification of artifacts in magnetoencephalographicrecordings, In Jordan MI, Kearns MJ, and Solla SA, editors, Advances in neural information processing systems,(10),22935. Cambridge, MA: MIT Press, 1997.

[23]. LanlanYu, EEG De-Noising Based on Wavelet Transformation, Bioinformatics and Biomedical Engineering, 2009. ICBBE 2009. 3rd International Conference, vol., no., pp.1-4, 11-13 June 2009.

\section{BIOGRAPHIES OF AUTHORS}

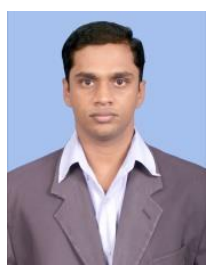

R.Suresh Kumar has completed his B.E. Electronics and Communication Engineering in the year 2004 from Thanthai Periyar Government Institute of Technology, Vellore. He received his M.E. degree (Applied Electronics) in the year 2007 from PSG College of Technology, Coimbatore. Currently he is doing his research at Karpagam University, Coimbatore. His areas of interest are VLSI and signal processing.

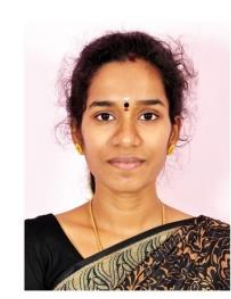

P.Manimegalai has completed her B.E. Biomedical Instrumentation Engineering in the year 2000 from Avinashilingam University. She obtained her M.E Applied Electronics from Govt. College of Technology by 2008. She obtained her Ph.D. from Anna University, Chennai from 2013. Currently she is working as Professor in ECE Department at Karpagam University, Coimbatore. Her areas of interest are biosignal processing and image processing. She has published more than 20 national and international journals. 